\title{
Evaluación de lesiones de la columna cervical en pacientes con politraumatismos, en el Servicio de Urgencias
}

\author{
Sofía Frank, Romina Cavallaro, Sergio Sánchez, Alberto Tulli, Alejandro Griglio, Federico Koll \\ Servicio de Ortopedia y Traumatología. Hospital Interzonal General de Agudos “Dr. José Penna”, Bahía Blanca, \\ Buenos Aires, Argentina
}

\section{RESUMEN}

Introducción: Tradicionalmente se ha recurrido al examen clínico-neurológico y a los estudios por imágenes para identificar lesiones de la columna cervical en pacientes con politraumatismos. En diferentes centros de trauma, la evaluación inicial de estos pacientes incluye un examen clínico-neurológico más radiografías obligatorias, independientemente de una semiología normal, en un paciente despierto y consciente. Esto se debe a la creencia de que el examen clínico es insuficiente para detectar estas lesiones. Materiales y Métodos: Se trata de un estudio transversal y observacional para determinar la sensibilidad y la especificidad de la evaluación clínica para el diagnóstico de lesiones de la columna cervical comparadas con las de los estudios por imágenes, en un período de 22 meses, en un Servicio de Urgencias. Resultados: Durante este período, se asistió a 127 pacientes. El examen físico neurológico fue normal en 101 pacientes, ninguno tenía una lesión constatada en las radiografías o las imágenes tomográficas. Veintiséis pacientes refirieron dolor a la palpación de las apófisis espinosas; en 6 de ellos, se constató una lesión de la columna cervical con la tomografía. La sensibilidad del examen físico fue del $100 \%$ y la especificidad, del $83 \%$. La tasa de falsos positivos fue del 17\% y no se registró ningún falso negativo. Conclusiones: Los datos de este estudio demuestran que la mayoría de los estudios por imágenes, radiografías o tomografías computarizadas podrían disminuirse, sin perder la sensibilidad del diagnóstico de lesiones de la columna cervical, reduciendo así los costos de la atención.

Palabras clave: Pesquisa; lesiones; columna cervical; politraumatismos; sensibilidad; especificidad. Nivel de Evidencia: IV

\section{Evaluation of Cervical Spine Injuries in Polytraumatized Patients, in Emergency Service}

\begin{abstract}
Introduction: The screening for cervical spine injuries in polytraumatized patients has traditionally consisted in neurological clinical examination and radiographic evaluation. In different trauma centers, the initial evaluation of these patients, being awake and conscious, includes neurological clinical examination and radiographs, even if they present a normal semiology. This is due to the belief that clinical examination is insufficient for the detection of these lesions. Materials and Methods: This is a cross-sectional and observational study to determine the sensitivity and specificity of the clinical examination for the diagnosis of cervical spine injuries, comparing imaging studies over a 22-month period in an emergency unit. Results: During this period, 127 patients were assisted. In 101 patients, the neurological and physical examination was normal. No injuries were recorded when performing radiographs or CT scans on these patients. During examination, 26 patients felt pain after palpation of the spinous processes. After a CT scan, 6 of these patients were found to have a cervical spine injury. The sensitivity of the physical examination was $100 \%$ and the specificity was $83 \%$. The false positive rate was $17 \%$ and no false negatives were recorded. Conclusions: The data from this study shows that the majority of studies by imaging, radiographs or CT scans could be reduced, without losing the sensitivity of cervical spine injuries diagnoses, and thus reducing the costs of care.
\end{abstract}

Key words: Screening; cervical spine injury; polytraumatized; sensitivity; specificity.

Level of Evidence: IV

Recibido el 2-5-2020. Aceptado luego de la evaluación el 29-7-2020 • Dra. SOFÍA FRANK • sofifrank_04@ hotmail.com ID https://orcid.org/0000-0001-9377-263X

Cómo citar este artículo: Frank S, Cavallaro R, Sánchez S, Tulli A, Griglio A, Koll F. Evaluación de lesiones de la columna cervical en pacientes con politraumatismos en el Servicio de Urgencias. Rev Asoc Argent Ortop Traumatol 2021;86(1):71-76. https://doi.org/10.15417/issn. 1852-7434.2021.86.1.1111 


\section{INTRODUCCIÓN}

Se considera que un paciente tiene politraumatismos si presenta una o varias lesiones de origen traumático, de las cuales, al menos una, puede poner en riesgo la vida, de forma más o menos inmediata. ${ }^{1}$ Suele tratarse de una persona de cualquier edad que sufre una lesión traumática externa habitualmente de alta energía. Tradicionalmente, el método para identificar las lesiones de la columna cervical en pacientes con politraumatismos ha sido el examen clínico semiológico, que se complementa con la evaluación radiográfica. Existen reglas de predicción clínica para ayudar a los profesionales a mejorar la toma de decisiones respecto del uso de estudios por imágenes para diagnosticar este tipo de lesiones. La Regla Canadiense de la Columna Cervical (Canadian C-Spine Rule) ha tenido una sensibilidad del $100 \%$ y una especificidad del $42,5 \%$ para descartar lesiones cervicales en pacientes estables y conscientes con traumatismos de la columna cervical. ${ }^{2}$ Se basa en tres criterios de alto riesgo: edad $>65$ años, mecanismos peligrosos y parestesias en extremidades; cinco criterios de bajo riesgo: peatón atropellado por un vehículo de motor, permanecer sentado o deambular, dolor de cuello de inicio tardío y ausencia de dolor en la línea media del cuello. El último criterio de bajo riesgo es la posibilidad de rotar el cuello a $45^{\circ}$. Si el paciente tiene algún criterio de alto riesgo, es preciso tomarle radiografías. Si tiene criterios de bajo riesgo y puede rotar el cuello, podría omitirse este estudio. Como esta regla se basa en el uso de imágenes en todos los pacientes que tengan resultados positivos, la baja especificidad y la alta tasa de falsos positivos indicarían que un gran cantidad de pacientes sin lesiones serían sometidos a estudios innecesarios. ${ }^{3}$ Grandes programas de implementación multicéntricos confirman la seguridad y el efecto clínico de esta herramienta para la pesquisa de lesiones de la columna cervical en pacientes alertas y estables. A pesar de esta evidencia, en diferentes centros, la evaluación inicial de estos pacientes incluye el examen clínico del cuello más radiografías obligatorias, al margen de un examen con semiología normal, en un paciente despierto y consciente. Esto se debe a la creencia de que el examen clínico es insuficiente para detectar lesiones de la columna cervical. El primer objetivo de este estudio fue describir y analizar la eficacia del examen clínico neurológico de la columna cervical en pacientes con politraumatismos conscientes y reactivos; el segundo objetivo fue compararlo con los estudios de imágenes realizados.

\section{MATERIALES Y MÉTODOS}

Este estudio tiene un diseño transversal y observacional, y se llevó a cabo en un único centro médico. Los datos se recolectaron de manera prospectiva, desde diciembre de 2016 hasta octubre de 2018, en el Servicio de Urgencias de nuestro Hospital Regional. Los criterios de inclusión fueron: pacientes con politraumatismos, $>18$ años, puntaje de Glasgow $\geq 14$ y estables. Se excluyó a pacientes inconscientes o intoxicados, embarazadas y a aquellos que tenían algún vínculo familiar con los investigadores. Este estudio fue aprobado por el Comité de Ética del Hospital. Cada paciente otorgó su consentimiento informado antes de incorporarse en el estudio.

Si el paciente no tenía dolor espontáneo o a la palpación, parestesias o paresias durante el examen físico, se le retiraba el collar cervical y se le ordenaba hacer movilidad activa, tanto en rotación, como en flexión y extensión. Independientemente de la semiología y con fines de estudio, a todos se les tomaron radiografías de la columna cervical lateral. Cuando las radiografías fueron dudosas o había sospecha clínica de lesión ósea en la columna cervical, se realizó una tomografía computarizada. Si el paciente sufría dolor a la palpación o con los movimientos, se daba por terminado el examen y se volvía a colocar el collar. En esta situación, la columna cervical no se consideraba indemne y se tomaron imágenes. Si no se observaban anomalías y el nuevo examen físico era normal, la columna cervical se consideraba libre de lesiones y se quitaba el collar (Figura 1).

Las variables estudiadas fueron: datos demográficos completos, edad, sexo, tipo de traumatismo y mecanismo de la lesión. En la cinemática del trauma, se agruparon las siguientes categorías: accidentes vehiculares de motocicleta o automotor, intento de suicidio, caída de altura, aplastamiento, accidentes deportivos y accidentes de peatón o bicicleta. Se registraron los datos del examen físico completo, el examen neurológico y los hallazgos en los estudios por imágenes. Las imágenes fueron definidas como patológicas o normales según la presencia o no de lesiones óseas visibles agudas, y fueron evaluadas por dos médicos especialistas. 


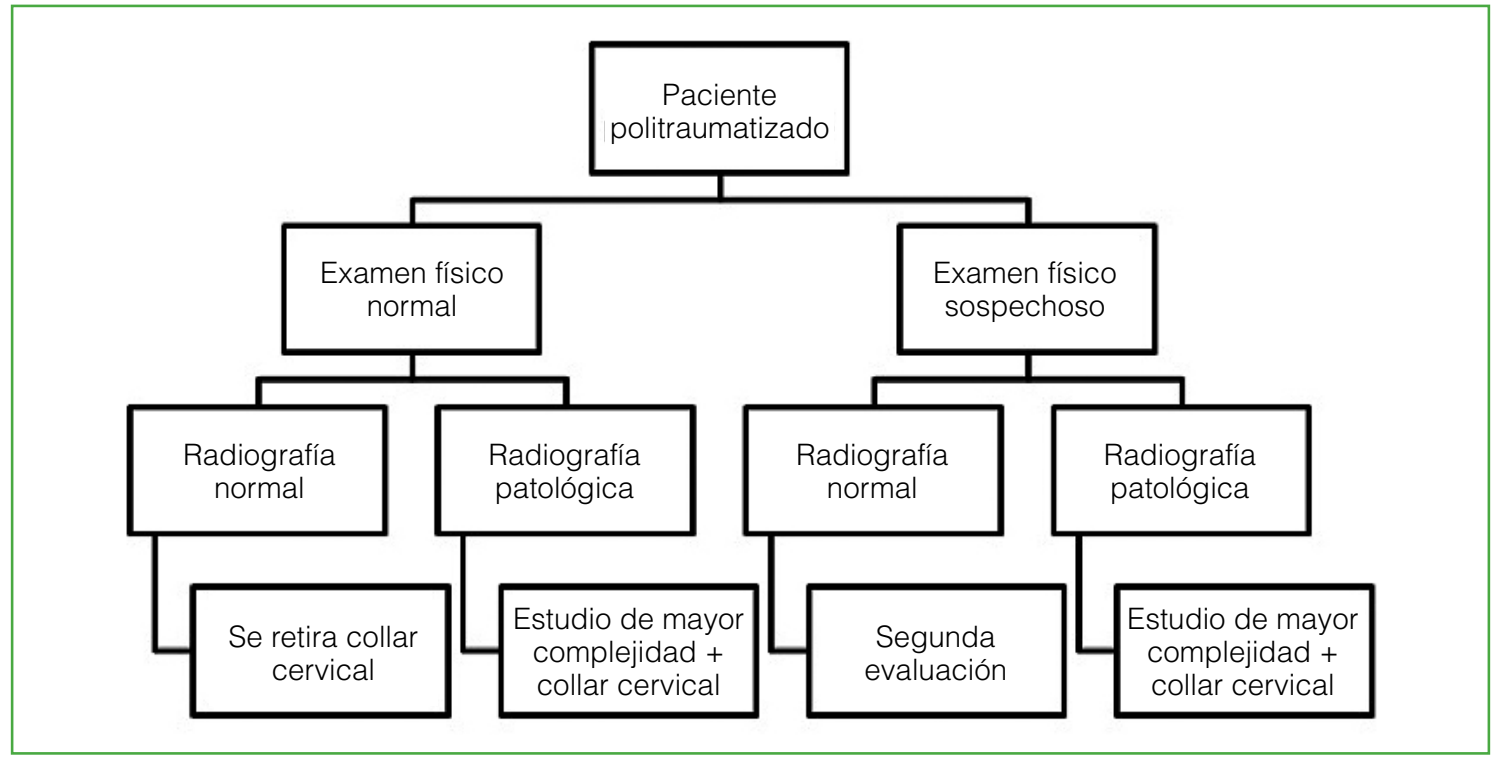

Figura 1. Protocolo de manejo del paciente politraumatizado.

\section{RESULTADOS}

Durante el período de estudio, se asistió a 127 pacientes con politraumatismos, despiertos y conscientes. El 59\% eran hombres. La edad media era de 35 años. La causa más frecuente de los politraumatismos fue el accidente vehicular, seguida de la caída de altura (Figura 2). Treinta y tres pacientes tenían una o más lesiones asociadas (Figura 3). La lesión asociada más frecuente fue la fractura de cúbito y radio, seguida de la fractura de tibia y peroné, y la de dos o más costillas. A todos se les realizó un examen físico sistematizado del cuello y se les tomaron radiografías. El examen físico de la columna cervical fue normal en 101 pacientes, ninguno tenía una lesión constatada con imágenes.

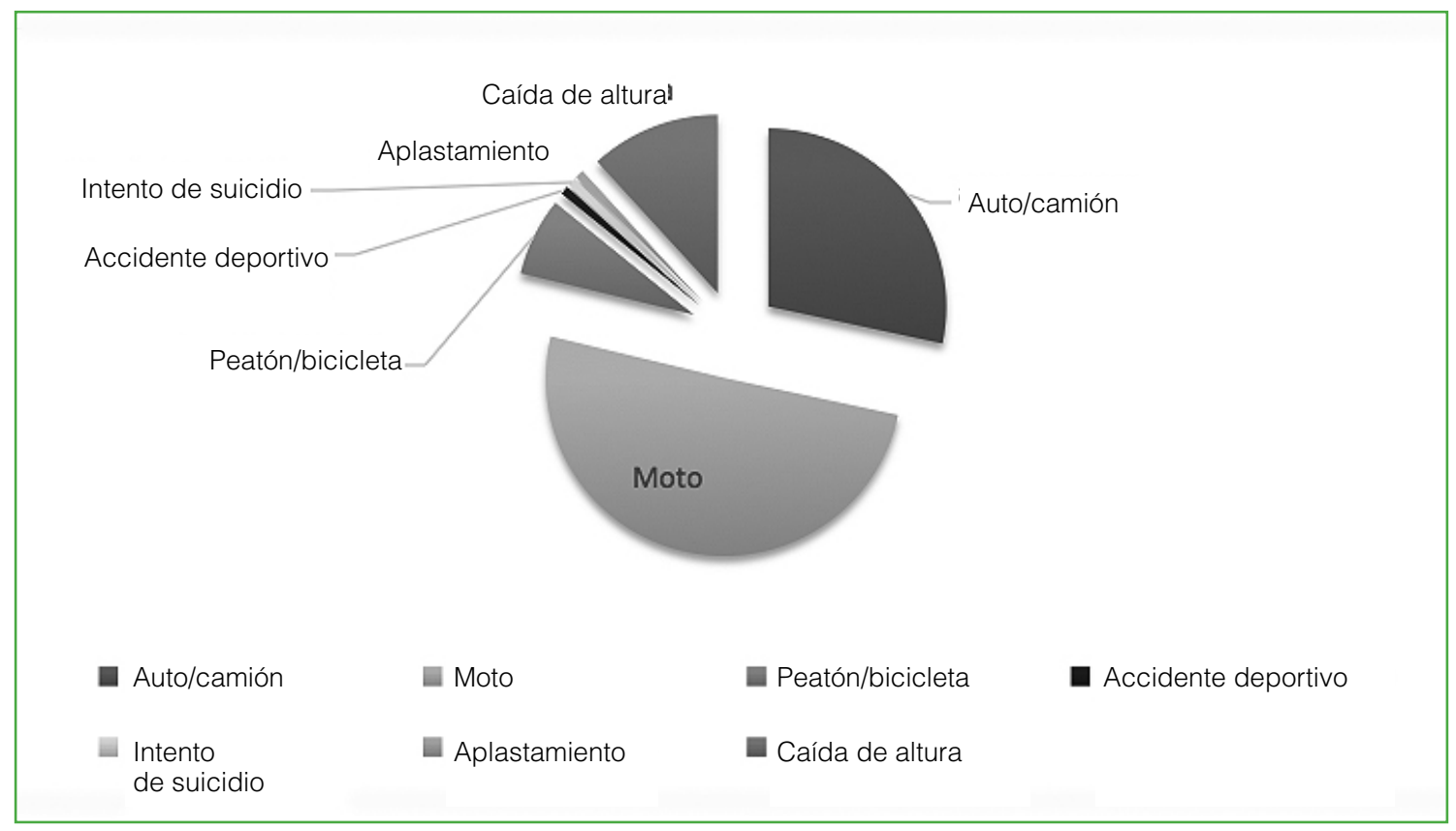

Figura 2. Mecanismo de la lesión. 


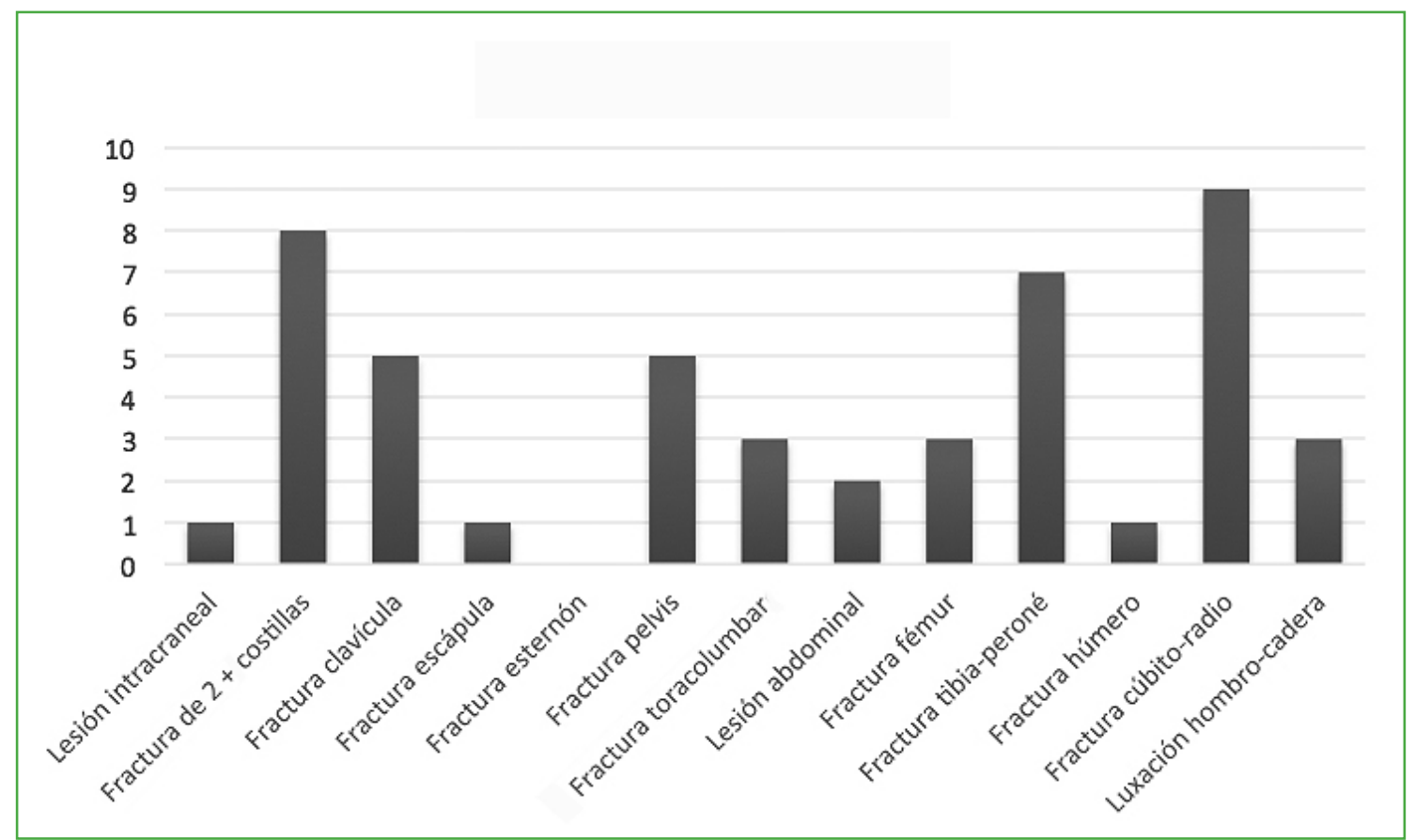

Figura 3. Lesiones asociadas.

Veintiséis sintieron dolor durante el examen del cuello; en seis de ellos, se constató una lesión ósea de la columna cervical (Tabla), ninguno sufrió una lesión neurológica asociada según la clasificación ASIA (American Spinal Injury Association). La sensibilidad del examen físico para diagnosticar lesiones de la columna cervical fue del $100 \%$ y la especificidad, del $83 \%$. La tasa de falsos positivos para el diagnóstico fue del 17\% y no se registró ningún falso negativo. Se calculó un valor predictivo negativo del 100\%. Cuatro pacientes tenían lesiones de tipo A de la Clasificación AOSpine para lesiones subaxiales, y recibieron tratamiento conservador, uno había sufrido una fractura de tipo IIIB de C1, que fue tratada con halo-chaleco y otro, una fractura de tipo B2 de la Clasificación AOSpine de columna subaxial, que fue tratada con artrodesis posterior instrumentada.

Tabla. Examen semiológico vs. examen radiológico de la muestra

$\begin{array}{lccc} & \text { Radiografia normal } & \text { Radiografía patológica } & \text { Total } \\ \text { Semiología normal } & 101 & 0 & 101 \\ \text { Semiología sospechosa } & 20 & 6 & 26 \\ \text { Total } & 121 & 6 & 127\end{array}$

\section{DISCUSIÓN}

La incidencia de fracturas de la columna cervical en los pacientes con politraumatismos es relativamente baja. En esta serie, fue del 4,7\%. Este dato coincide con las tasas publicadas que varían del 2,8\% al 7,7\%. ${ }^{4}$ En este estudio, se demostró que el examen clínico del cuello tiene una alta sensibilidad para el diagnóstico de lesiones de la columna cervical. Al igual que esta serie, numerosas publicaciones han documentado que el examen clínico sistematizado es una herramienta extremadamente sensible para la pesquisa de lesiones en pacientes vigiles y alertas..$^{5-9}$ 
Pese a esto, se toma gran cantidad de radiografías innecesarias en los servicios de urgencias. En este caso, el 94\% de las imágenes no reveló lesiones óseas, porcentaje similar a lo ya publicado, lo que demuestra que más del 98\% de las imágenes no muestran fracturas. ${ }^{10}$ Este bajo rendimiento se asocia a costos significativos y un mal uso de los recursos del sistema de salud, además de que los pacientes son expuestos a radiaciones potencialmente peligrosas sin indicación clínica o a inmovilizaciones prolongadas. A pesar de la evidencia disponible, entre los profesionales, el criterio es muy variable respecto al uso de las radiografías de columna cervical. Esto podría deberse a las implicancias clínicas y medicolegales de no diagnosticar una lesión de columna. Quizás esta sea la razón del uso excesivo de los estudios por imágenes en los servicios de urgencias. ${ }^{11}$ El examen clínico y las reglas de predicción, como la Canadian C-Spine Rule, ${ }^{12}$ se deberían poder utilizar para evaluar a pacientes con politraumatismos, vigiles y sin deterioro neurológico, a fin de determinar si son necesarios los estudios por imágenes complementarios. De esta manera, se podría brindar una atención segura, basada en la evidencia y costo-eficaz a los pacientes con politraumatismos.

Este estudio es el único informe prospectivo sobre la evaluación de lesiones de la columna cervical en pacientes con politraumatismos publicado en nuestro país. Las limitaciones de esta serie son: el tamaño de la muestra y la cantidad de lesiones cervicales halladas. Por otro lado, este estudio no examina las lesiones disco-ligamentarias aisladas sin fractura o luxación, lo que podría constituir un sesgo. No obstante, las lesiones no esqueléticas de la columna cervical son poco frecuentes, según lo publicado. ${ }^{13}$

\section{CONCLUSIONES}

Los datos de este estudio demuestran que la evaluación clínica y sistematizada de la columna cervical tiene una alta sensibilidad para el diagnóstico de lesiones óseas en la columna de pacientes conscientes y respondedores. Por lo tanto, se podría evitar la mayoría de los estudios por imágenes y así disminuir los costos de la atención y la radiación innecesaria para los pacientes. Es necesario llevar a cabo más estudios que permitan establecer el diagnóstico preciso de estas lesiones con el objetivo ulterior de mejorar la atención y reducir los costos de la asistencia de pacientes con politraumatismos.

Conflicto de intereses: Los autores no declaran conflictos de intereses.

\section{BIBLIOGRAFÍA}

1. Vegas Rodríguez FJ, Caballero Trenado JV. Valoración del paciente politraumatizado. Bajadoz: Gerencia del Área de Salud de Bajadoz; 2016:1-16. Disponible en: https:/www.areasaludbadajoz.com/images/stories/politraumatizado.pdf

2. Stiell IG, Clement CM, McKnight RD, Brison R, Schull MJ, Rowe B, et al. The Canadian C-spine rule versus the NEXUS low-risk criteria in patients with trauma. N Engl J Med 2003;349(26):2510-8. https://doi.org/10.1056/NEJMoa031375

3. Michaleff ZA, Maher CG, Verhagen AP, Rebbeck T, Lin CW. Accuracy of the Canadian C-spine rule and NEXUS to screen for clinically important cervical spine injury in patients following blunt trauma: a systematic review. CMAJ 2012;184(16):E867-E876. https://doi.org/10.1503/cmaj.120675

4. Stiell IG, Wells GA, Vandemheen KL, Clement CM, Lesiuk H, De Maio VJ, et al. The Canadian C-spine rule for radiography in alert and stable trauma patients. JAMA 2001;286(15):1841-8. https://doi.org/10.1001/jama.286.15.1841 
5. Stiell IG, Clement CM, Lowe M, Sheehan C, Miller J, Armstrong S, et al. A multicenter program to implement the Canadian C-Spine rule by emergency department triage nurses. Ann Emerg Med 2018;72(4):333-41. https://doi.org/10.1016/j.annemergmed.2018.03.033

6. Rose MK, Rosal LM, Gonzalez RP, Rostas JW, Baker JA, Simmons JD, et al. Clinical clearance of the cervical spine in patients with distracting injuries: It is time to dispel the myth. J Trauma Acute Care Surg 2012;73(2):498502. https://doi.org/10.1097/ta.0b013e3182587634

7. Paykin G, O’Reilly G, Ackland HM, Mitra B. The NEXUS criteria are insufficient to exclude cervical spine fractures in older blunt trauma patients. Injury 2017;48(5):1020-4. https://doi.org/10.1016/j.injury.2017.02.013

8. Vaillancourt C, Stiell IG, Beaudoin T, Maloney J, Anton AR, Bradford P, et al. The out-of-hospital validation of the Canadian C-Spine Rule by paramedics. Ann Emerg Med 2009;54(5):663-71.e1. https://doi.org/10.1016/j.annemergmed.2009.03.008

9. Coffey F, Hewitt S, Stiell I, Howarth N, Miller P, Clement C, et al. Validation of the Canadian c-spine rule in the UK emergency department setting. Emerg Med J 2011;28(10):873-6. https://doi.org/10.1136/emj.2009.089508

10. Duane TM, Wilson SP, Mayglothling J, Wolfe LG, Aboutanos MB, Whelan JF, et al. Canadian Cervical Spine rule compared with computed tomography: a prospective analysis. J Trauma 2011;71(2):352-7. https://doi.org/10.1097/TA.0b013e318220a98c

11. Moser N, Lemeunier N, Southerst D, Shearer H, Murnaghan K, Sutton D, et al. Validity and reliability of clinical prediction rules used to screen for cervical spine injury in alert low-risk patients with blunt trauma to the neck: part 2. A systematic review from the Cervical Assessment and Diagnosis Research Evaluation (CADRE) Collaboration. Eur Spine J 2018;27(6):1219-33. https://doi.org/10.1007/s00586-017-5301-6

12. Rethnam U, Yesupalan R, Gandham G. Does applying the Canadian Cervical Spine rule reduce cervical spine radiography rates in alert patients with blunt trauma to the neck? A retrospective analysis. BMC Med Imaging 2008;8:12. https://doi-org/10.1186/1471-2342-8-12

13. Ecker TM, Kleinschmidt M, Martinolli L, Zimmermann H, Exadaktylos AK. Clinical presentation of a traumatic cervical spine disc rupture in alpine sports: a case report. Scand J Trauma Resusc Emerg Med 2008;16:14. https://doi.org/10.1186/1757-7241-16-14 